\title{
The Application of Visualization in Optimizing Personalized Recommendation Result
}

\author{
Dong Qian, Weiwei Jia \\ Communication University of China, China \\ *qiandong1109@163.com
}

Keywords: visualization; personalized recommendation; user interaction; user experience

\begin{abstract}
With the explosive growth of big data, people want more efficient access to their most relevant information. The personalized recommendation system can pick individual interested items and send it to the specific user. However, the traditional recommendation result are generally showed by images or a simple list without any feedback, which is not good for user experience and result optimization. This paper uses the visualization technologies to visualize the recommendation result and provides interactive controls for users. Then the interaction data would be input for the next round of recommendation. In the end, we give a user study to test our optimization effect.
\end{abstract}

\section{Introduction}

The personalized recommendation algorithms are based on big datasets and compute the most relevant information for the current user and offer the user's most interested items. Obviously, users are the core of personalized recommendation system. Traditional methods to show the recommendation result are mostly based on a simple list or some static images. However, this one-way data display merely make the users accept the result without any feedback and interaction control. As a result, users are isolated to the recommendation system.

The visualization technology transform boring data to dynamic graphics or animations by a series of computing process. The visualization can assist users in finding potential relationships among data and mining hidden models from information seemingly irrelevant.

In this paper, we applicate the visualization technology in result display of personalized recommendation to optimize user experience as well as the recommended accuracy. We add various interactive controls to our visualization and record the interaction data for the next round of recommendation. What's more, we do a user study to test the optimizing effect of our methods.

\section{Related Work}

There have been some researches about the combination of recommendation and visualization technologies.

PeerChooser [1] uses graphs to represent user relations to provide a visual explanation of the recommendation process, and users can tweak neighborhood in the graph. SmallWorlds [2] constructs a visual interactive interface to recommend friends and items based on the Facebook API. They try to make the recommendation process transparency for users. SFViz [3] gives a visual recommender system which facilitates exploration based on social tags. The system support users to seek potential friends by multiscale aggregations through the recommendation process. TasteWeights [4] presents an interactive recommendation system which allows user to manipulate the importance of friends in social network and generates corresponding recommendation.

However, all researches above are try to visualize the recommendation process, but our work focus on visualization for the recommendation result. There are two considerations for this. Firstly, the item set before input to the recommender system is very complex and large, so visualizing all the items on screen may mislead users and put negative effect on data understanding. Secondly, the visualization 
for result can provide users with interaction controls and send the feedback to recommendation algorithms for prediction accuracy improvement.

\section{Visualization Process}

Generally, our system can be divided into three modules: Recommendation algorithm module, Result visualization module and Interaction feedback module, as shown in Fig. 1.

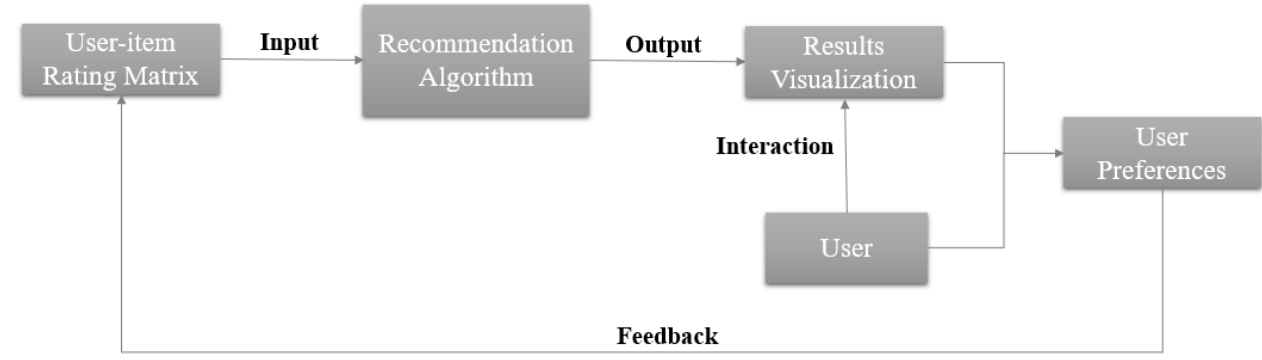

Figure 1. The processes of signature and authentication in digital signature system

\subsection{Recommendation Algorithm.}

In the recommendation algorithm module, we mainly use a collaborative filtering recommendation algorithm [5]. It finds a nearest user or item set for the target user and computes the users' similarity as rating weight to give the recommendation.

There are three common similarity measure methods: Cosine similarity [6], Euclidean distance [7] and Pearson similarity [8]. The cosine similarity measure treats user ratings as a space vector and pays more attention to the differences between dimensions rather than values. Euclidean distance computes the direct space distance, but it would cause big error in similarity measure for multi-dimensional variables. Pearson similarity is based on the joint ratings by users. We test our system on Movielens dataset. As the multivariate nature of movie items, we choose Pearson similarity measure for our collaborative filtering algorithm.

\subsection{Result Visualization and Interaction}

We visualize the result by D3.js. The force-directed layout is a simulation of physical particles and fits to describe relations among many nodes.

Considering we try to make user understand and control the similarity between movie items, each movie can be represented as a node and the edges can indicate the similarity weight between movies. We visualize movies as circles while genres as rectangles. The rating scores are mapping to the node radius and colors. A lower score would lead to a smaller and greater transparency circle node. We map the movie numbers of a genre to the rectangle area with a directly proportional relationship. The movie titles and genre names are shown as labels of nodes. Our visualization interface is shown in Fig. 2 .

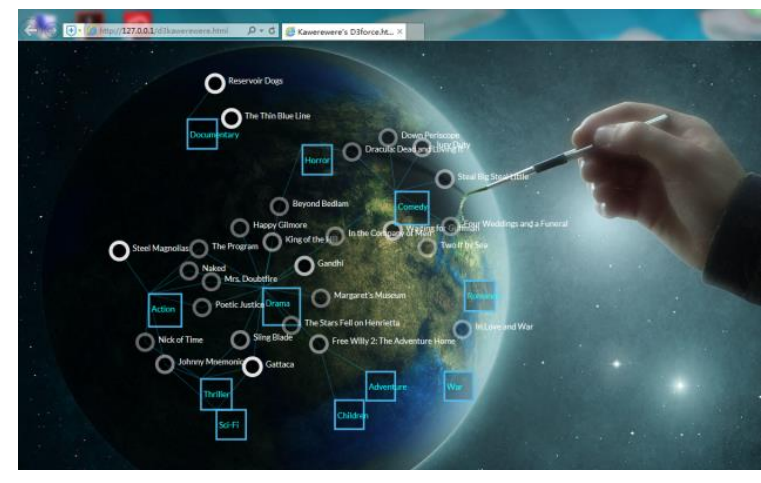

Figure2. Result visualization interface

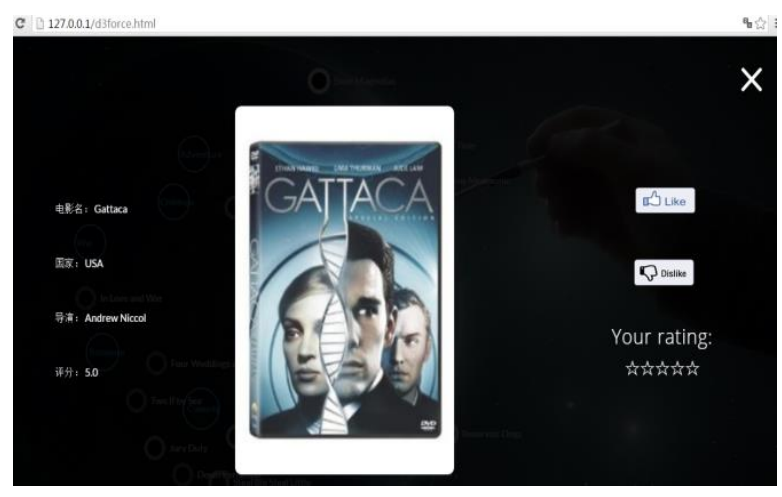

Figure 3. An example page for movie details 
We add the drag, zoom and pan interactions to facilitate user exploration. Users can drag a node and fix its position. The area and label of the hovered node would be larger at the same time. Moreover, users can also click on a movie node and browse the detailed information. The additional information such as the poster picture and director, country of the selected node are shown in the detailed page. An example of the detailed page is shown in Fig. 3.

There are two buttons and a rating panel on the right side of the details page. If the user clicks the "Like" button, label of the movie would be highlighted as red color after the details page closed and its transparency would no longer be reduced. However, the movie node would be deleted from the visualization interface once user click the "Dislike" button.

User can give a new rating score to the movie on the rating panel. We record the new rating data and save it into a local file which includes the user id, movie id and rating score. The recorded data would be added to the user preference dataset for the next round of recommendation.

\section{User study}

To verify the effect of result visualization, we carried out a user experience test. We selected five users with difference movie tastes as the test users. Then we selected twenty movies with different types from the base-set for rating. If the user had watched the test movie, he can rate according to his preferences. Otherwise he can give an expectation score on the basis of movie information from IMDB website.

We added the test users' rating data to base-set and inputted the base-set into user-based collaborative filtering recommendation algorithm for the first round of calculation. Firstly, we set the number of recommended movies to 20 . Then, test users manipulated the result in the visualization interface and give their feedback. Their interaction data was added to the next round of recommendation. We recorded the number of movies which had be labeled as "Like" to represent user satisfaction with the result of each round of recommendation. We made five rounds of recommendation in the test and plot a user satisfaction comparison chart as shown in Fig. 4.

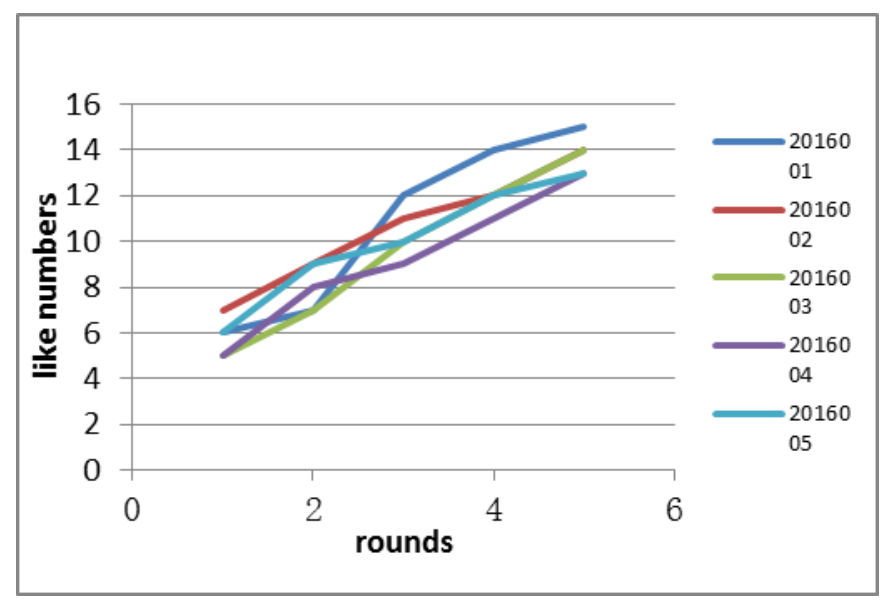

Figure 4. User satisfaction comparison of the five rounds of recommendation

It can be seen that there are very few movies labeled "Like" in the first round of recommendation because we merely counted 20 rating items for each user in the first time. Hence, the recommendation result was poor when there are little user preferences data. We can clearly see that from the second round of recommendation, the user satisfaction on the recommendation result is higher than the previous round. We indicate that the user satisfaction increased with more interaction data from each round added to the base-set. This result matches the conclusion from our comparison experiment between item-based and user-based collaborative filtering algorithm. 


\section{Conclusions}

In this paper, we study the application of visualization in optimizing the personalized recommendation result. We use the user-based collaborative filtering algorithm to compute the recommendation. We construct a visualization interface to display the result and provide many interaction controls for users. The interaction data would be recorded and inputted to the next round of recommendation. In the end, we conduct a user study about the optimization effect. The results show that with the increase of interactions, user satisfaction would gradually improves. In the future work, we would try different types of datasets and add more interactions to further enhance the user experience.

\section{Acknowledgment}

The work is supported by grants from National Science and Technology Supporting Program of China (2014BAH10F00), New Century Excellent Talents in University of Ministry of Education of China (NCET-12-0944), University Research Program of Communication University of China (3132015XNG1522). We thank the reviewers and editors for their helpful comments.

\section{References}

[1] O'Donovan J, Smyth B, Gretarsson B, et al. PeerChooser: visual interactive recommendation[C]// Conference on Human Factors in Computing Systems, CHI 2008, 2008, Florence, Italy, April. 2008:1085--1088.

[2] Brynjar G, John O, Svetlin B, et al. Smallworlds: visualizing social recommendations[J]. Computer Graphics Forum, 2010, 29(3):833-842.

[3] Gou L, You F, Guo J, et al. SFViz: Interest-based friends exploration and recommendation in social networks[C]// Proceedings of the 2011 Visual Information Communication - International Symposium. ACM, 2011:1-10.

[4] Bostandjiev S, O'Donovan J, Llerer T. TasteWeights: a visual interactive hybrid recommender system[C]// ACM Conference on Recommender Systems. ACM, 2012:35-42

[5] Sarwar, Badrul, Karypis, et al. Item-based collaborative filtering recommendation algorithmus[C]// 2001:págs. 8-8.

[6] Liu Y, Qin Y. Cosine Similarity-based m-class Classifier and Algorithm[J]. Computer \& Digital Engineering, 2014.

[7] Dapeng H, Lin Q, Zhou J. An Improved Similarity Algorithm for Personalized Recommendation[C]// International Forum on Computer Science-Technology and Applications. IEEE, 2009:54-57.

[8] Wu W J, Xu Y. Correlation analysis of visual verbs' subcategorization based on Pearson's correlation coefficient.[C]// International Conference on Machine Learning and Cybernetics, ICMLC 2010, Qingdao, China, July 11-14, 2010, Proceedings. 2010:2042-2046. 